\title{
Genomic Tandem Quadruplication is Associated with Ketoconazole Resistance in Malassezia pachydermatis ${ }^{\mathrm{S}}$
}

\author{
Minchul Kim ${ }^{1 \dagger}$, Yong-Joon $\mathrm{Cho}^{2 \dagger}$, Minji Park ${ }^{1}$, Yoojeong Choi ${ }^{1}$, Sun Young Hwang ${ }^{3}$, and Won Hee Jung ${ }^{1 *}$ \\ ${ }^{1}$ Department of Systems Biotechnology, Chung-Ang University, Anseong 17546, Republic of Korea \\ ${ }^{2}$ Korea Polar Research Institute, Incheon 21990, Republic of Korea \\ ${ }^{3}$ Haemaru Small Animal Research Institute, Sungnam 13590, Republic of Korea
}

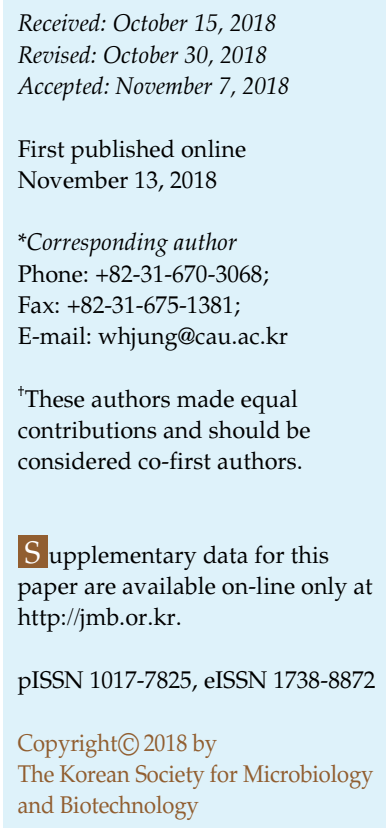

Malassezia pachydermatis is a commensal yeast found on the skin of dogs. However, M. pachydermatis is also considered an opportunistic pathogen and is associated with various canine skin diseases including otitis externa and atopic dermatitis, which usually require treatment using an azole antifungal drug, such as ketoconazole. In this study, we isolated a ketoconazole-resistant strain of M. pachydermatis, designated "KCTC 27587," from the external ear canal of a dog with otitis externa and analyzed its resistance mechanism. To understand the mechanism underlying ketoconazole resistance of the clinical isolate M. pachydermatis KCTC 27587, the whole genome of the yeast was sequenced using the PacBio platform and was compared with $M$. pachydermatis type strain CBS 1879. We found that a $\sim 84-\mathrm{kb}$ region in chromosome 4 of $M$. pachydermatis KCTC 27587 was tandemly quadruplicated. The quadruplicated region contains 52 protein coding genes, including the homologs of ERG4 and ERG11, whose overexpression is known to be associated with azole resistance. Our data suggest that the quadruplication of the $\sim 84-\mathrm{kb}$ region may be the cause of the ketoconazole resistance in M. pachydermatis KCTC 27587.

Keywords: Malassezia pachydermatis, genomic quadruplication, ketoconazole, antifungal, resistance, dog

\section{Introduction}

Malassezia species are basidiomycetous fungi that are commonly found on the skin of various animals and are considered a residential cutaneous fungal flora. Among this species, Malassezia pachydermatis is a normal organism in dogs and is found in the ear canals, on the lips, axillae folds, anal sacs, rectum, and interdigital spaces. However, M. pachydermatis can cause diseases such as otitis externa and dermatitis in dogs with compromised immunity or altered skin condition [1, 2]. In particular, comparison of the mycobiota in the ear canals of healthy dogs and dogs with otitis externa based on sequencing data showed an extended population of M. pachydermatis in dogs with the disease and confirmed the association of M. pachydermatis with this major skin disease in dogs [3]. Although $M$. pachydermatis is primarily zoophilic [4], the yeast frequently colonizes the human skin, of neonates in particular, and causes systemic infections $[5,6]$.

Ketoconazole, an antifungal azole derivative, is one of the drugs the most commonly used to treat canine otitis externa and dermatitis caused by $M$. pachydermatis $[4,7,8]$. In model fungi such as Sacharomyces cerevisiae and Candida albicans, ketoconazole binds to lanosterol 14- $\alpha$-demethylase encoded by ERG11 and inhibits the synthesis of ergosterol, which is a major constituent of the fungal membrane [9, 10]. Most $M$. pachydermatis strains are susceptible to ketoconazole; however, the occurrence of resistant strains has been reported [11, 12]. To date, only one study has investigated the mechanisms of azole resistance in M. pachydermatis [13]. The study suggested an involvement of drug efflux pumps [13]; however, it used drug efflux pump inhibitors, not a molecular genetic approach.

In the current study, we isolated M. pachydermatis strains 
from dogs with various skin diseases, including otitis externa, and the susceptibility of the fungi to ketoconazole was determined. Whereas most of the isolates were susceptible to ketoconazole, three strains showed significantly increased minimal inhibitory concentrations (MICs) implicating that they are resistant to the drug. The strain that displayed the highest MIC was selected for genetic analysis to elucidate its ketoconazole resistance mechanism. Our study revealed the existence of a tandem quadruplication in chromosome 4 of the resistant strain and showed that the quadruplicated region contains genes required for ergosterol synthesis. Moreover, transcriptome analysis suggested that the expression of genes in the quadruplicated region was strongly increased. Our results suggest that the chromosomal region that was quadruplicated in M. pachydermatis KCTC 27587 is important in ketoconazole resistance and imply that multiplication of the region contributes to ketoconazole resistance in M. pachydermatis.

\section{Materials and Methods}

\section{Clinical Sampling}

In total, 10 dogs with at least one of the clinical statuses as listed in Table 1, including Cushing's disease, pancreatitis, mass of cervix or face, heart disease, atopy, mass or cancer in the liver, and hypothyroidism, were presented at our veterinary hospital. The breeds of the dogs were Maltese, Shih-Tzu, Miniature Schnauzer, Poodle, and a mixed breed. Following routine clinical examination, the animals were aseptically swabbed in both ear canals or on a paw or other skin part according to a protocol approved by the Institutional Animal Care and Use Committees at Chung-Ang University (2014-00010). All dogs showed mild ear discharge, and we cleaned the ears rather than administrating antimicrobial or antifungal reagents. Swab samples were transported to the microbiology laboratory at ambient temperature and were processed within $1 \mathrm{~h}$ of collection.

\section{Isolation and Identification of M. pachydermatis}

Malassezia strains were grown in modified Dixon's (mDixon) medium ( $3.6 \%$ malt extract, $2 \%$ bile salt, $0.6 \%$ peptone, $1 \%$ Tween$40,0.2 \%$ oleic acid, $0.2 \%$ glycerol) at $34^{\circ} \mathrm{C}$ for 2 days [14]. To identify Malassezia isolates, a single colony was taken and suspended in chelating ion exchange resin solution $(20 \%(\mathrm{w} / \mathrm{v})$ Chelex (BioRad, USA) in Tris-ethylenediaminetetraacetic acid buffer) [15]. The mixture was boiled at $100^{\circ} \mathrm{C}$ for $20 \mathrm{~min}$, cooled, and centrifuged at $16,000 \mathrm{~g}$ for $5 \mathrm{~min}$. The supernatant containing genomic DNA was used for amplification of the D1/D2 region of the large subunit of rDNA by PCR using the universal primers NL-1 (5'-GCATAT CAATAAGCGGAGGAAAAG-3') and NL-4 (5'-GGTCCGTGT TTCAAGACGG-3') [16]. The PCR product was sequenced for species identification. Sequences from all 10 isolates obtained from each of the dogs showed more than $99 \%$ identity to that of the M. pachydermatis type strain CBS 1879 and thus, all 10 isolates were identified as M. pachydermatis. The identified M. pachydermatis strains were deposited to the Korean Collection for Type Culture (KCTC).

\section{Determination of Antifungal Susceptibility}

To evaluate the susceptibility of $M$. pachydermatis towards ketoconazole, minimal inhibitory concentrations (MICs) of the strains were determined using the modified broth serial dilution method based on the Clinical and Laboratory Standards Institute (CLSI) guideline [17]. A stock solution of ketoconazole was serially diluted two-fold in a total volume of $200 \mu \mathrm{l}$ of mDixon's medium in a 96-well plate. M. pachydermatis cells $\left(5 \times 10^{2}\right.$ to $2.5 \times$ $10^{3} \mathrm{CFU} / \mathrm{ml}$ ) were inoculated into each well of the 96 -well plate

Table 1. Ketoconazole sensitivity of clinical isolates.

\begin{tabular}{lllllc}
\hline \multicolumn{1}{c}{ Strains } & \multicolumn{1}{c}{ Source (Breed) } & Sex/Age & Isolation & Clinical diagnosis & MIC $(\mu \mathrm{g} / \mathrm{ml})$ \\
\hline CBS 1879 & Dog (unknown, CBS type strain) & Unknown & Ear & Otitis external & $0.25-0.5$ \\
KCTC 27575* & Dog (Maltese) & F/2 & Ear & Healthy & 0.5 \\
KCTC 27587 & Dog (Maltese) & F/11 & Ear & Otitis external, atopic dermatitis, food allergy & 8 \\
KCTC 27591 & Dog (Maltese) & M/9 & Paw & Cushing disease & 4 \\
KCTC 27593 & Dog (Maltese) & M/12 & Skin & Relapsed lymphoma & 4 \\
KCTC 27824 & Dog (mixed) & M/1 & Ear & Healthy & $0.125-0.25$ \\
KCTC 27825 & Dog (Maltese) & M/1 & Ear & Healthy & $0.125-0.25$ \\
KCTC 27826 & Dog (Poodle) & M/2 & Ear & Healthy & $0.25-0.5$ \\
KCTC 27827 & Dog (Shih-tzu) & M/1 & Ear & Healthy & 0.25 \\
KCTC 27828 & Dog (Maltese) & M/1 & Ear & Healthy & $0.125-0.5$ \\
KCTC 27829 & Dog (Miniature Schnauzer) & F(S) $/ 3$ & Ear & Nephrolithiasis & 0.25 \\
\hline
\end{tabular}

*KCTC: Korean Collection of Type Cultures

${ }^{q}$ Female (spayed) 
and incubated at $34^{\circ} \mathrm{C}$ for 2 days. MICs were determined as the lowest concentration at which there was no visible growth of the cells compared to that in medium without ketoconazole. MICs were determined at least three times independently.

\section{Genome Sequencing and Annotation}

M. pachydermatis KCTC 27587 was grown in mDixon medium at $34^{\circ} \mathrm{C}$ for 2 days, and genomic DNA was extracted from the cells as described previously [18]. The genomic DNA was fragmented using a g-TUBE device (Covaris, USA) to fragments of $20 \mathrm{~kb}$ in length, and a DNA library was constructed for the PacBio platform (PacBio, USA) according to the manufacturer's instructions. Sequencing was performed on the PacBio Sequel instrument, using one SMRT cell. The raw read sequences were assembled with canu v1.7 [19] with genomeSize set at 7.3 Mb. Contigs were polished using the Arrow consensus caller in PacBio SMRT Link v5.0.1. Telomeric motifs in chromosomal ends and mitochondrial contigs were reviewed by manual curation. Gene prediction was conducted with BRAKER v1.8 [20] and genes were annotated using eggNOG-mapper [21]. When the transcript expression was normalized to TPM (Transcripts Per Kilobase Million), the percentage of genes with TPM value over 10 in M. pachydermatis KCTC 27587 strain was $97.1 \%$ of total 4,116 genes. Genome sequencing data have been deposited to the Sequence Read Archive database of the National Center for Biotechnology Information (NCBI) under accession no. SRP156512.

\section{Transcriptome Analysis}

M. pachydermatis KCTC 27587 and CBS 1879 cells were incubated in mDixon's broth medium at $34^{\circ} \mathrm{C}$ for $6 \mathrm{~h}$ and harvested for RNA extraction. Total RNA was prepared using TransZolUp (Transgen Biotech, China), and the RNA integrity in each sample was evaluated using BioAnalyzer 2100 (Agilent Technologies, USA). Libraries for RNA-sequencing were constructed using the TruSeq Stranded Total RNA Sample Prep Kit (Illumina, USA) following the manufacturer's instructions. The libraries were sequenced on an Illumina HiSeq 2500 instrument (Illumina, USA) following the manufacturer's instructions, generating 75-bp paired-end reads for each sample. Raw sequence data were subjected to adapter sequence removal and quality-based trimming with Trimmomatic v. 0.36 using TruSeq adapter sequences [22]. Cleaned reads were mapped to the reference genome using bowtie 2 with default parameters settings [23]. FeatureCounts was used to count the reads mapped to each coding sequence [24]. The counts were transformed and normalized for differential expression analysis with DESeq2 [25]. The transcriptome data have been deposited to the Gene Expression Omnibus database of NCBI under accession no. GSE118354.

\section{Quantitative Real-Time PCR}

For the validation of gene copy numbers, quantitative real-time PCR (Q-RT-PCR) was conducted using genomic DNA as a template. Gene-specific primers for Q-RT-PCR were designed using Primer Express software v. 3.0 (Applied Biosystems, USA) and are listed in Table S1. Q-RT-PCRs were run on a 7500 RealTime PCR System (Applied Biosystems, Foster, USA). The relative copy number of each gene of interest was calculated by the $2^{-\Delta \Lambda C T}$ method [26]. The translation elongation factor 2 (TEF2) gene was used as an endogenous control for normalization.

\section{Generation of a Ketoconazole-Resistant M. pachydermatis Strain} by In Vitro Evolution

The ketoconazole-susceptible strain $M$. pachydermatis KCTC 27575 was used for in vitro evolution according to a previously reported method with modification [27]. The susceptible strain was periodically propagated in mDixon agar containing ketoconazole for seven transfers. For each transfer, a single colony was transferred to medium containing 2-fold serially increasing concentrations of ketoconazole (from $0.06 \mu \mathrm{g} / \mathrm{ml}$ to $8 \mu \mathrm{g} / \mathrm{ml}$ ) and incubated for 5 days at $34^{\circ} \mathrm{C}$ between transfers. Four independently generated resistant strains showing MICs equivalent to that of M. pachydermatis KCTC 27587 were obtained.

\section{Results and Discussion}

\section{Isolation of Ketoconazole-Resistant M. pachydermatis}

The MICs of 10 clinically isolated M. pachydermatis strains to ketoconazole were determined according to a modified CLSI broth microdilution method. The MICs of seven M. pachydermatis isolates, KCTC 27575, KCTC 27824, KCTC 27825, KCTC 27826, KCTC 27827, KCTC 27828, and KCTC 27829 , were between $0.125 \mu \mathrm{g} / \mathrm{ml}$ and $0.5 \mu \mathrm{g} / \mathrm{ml}$, which was the range for the ketoconazole-susceptible M. pachydermatis type strain, CBS 1879. However, three out of the 10 isolates, KCTC 27587, KCTC 27591, and KCTC 27593, showed significantly higher MICs of $4-8 \mu \mathrm{g} / \mathrm{ml}$, suggesting that they are resistant to ketoconazole (Table 1). Among the resistant strains, M. pachydermatis KCTC 27587, which was isolated from a dog suffering from otitis externa, atopic dermatitis, and food allergy, showed the highest MIC and was therefore selected for further investigation of the mechanism underlying ketoconazole resistance of M. pachydermatis.

\section{Whole Genome Sequencing of M. pachydermatis KCTC 27587}

To understand the mechanism of ketoconazole resistance of M. pachydermatis KCTC 27587, the whole genome of the yeast isolate was sequenced using the PacBio platform and compared with that of type strain $M$. pachydermatis CBS 1879. The genome and mitochondrial genome sizes of M. pachydermatis KCTC 27587 are 8,283,742 and 35,575 bp, respectively. In total, six chromosomes were assembled, and 4,116 protein-coding regions were predicted. The 
Table 2. Summary of the genome analysis.

\begin{tabular}{lcc}
\hline & \multicolumn{2}{c}{ PacBio } \\
\hline Reads & \multicolumn{2}{c}{138,081} \\
Read length (avg. bp) & \multicolumn{2}{c}{$7,310.2$} \\
Bases (Mb) & \multicolumn{2}{c}{$1,009.4$} \\
Coverage (fold) & \multicolumn{2}{c}{121.9} \\
\hline & Nuclear & Mitochondrial \\
\hline Total assembly size (bp) & $8,283,742$ & 35,575 \\
GC (\%) & 55.2 & 31.2 \\
Scaffold & 6 & 1 \\
Coding sequence & 4,116 & 18 \\
rRNA & 22 & 2 \\
tRNA & 77 & 19 \\
\hline
\end{tabular}

mitochondrial genome contains 18 coding regions (Table 2). Wu et al. recently analyzed the genome of the M. pachydermatis type strain CBS 1879 using Illumina HiSeq and found that it has a $8.2-\mathrm{Mbp}$ genome harboring 4,328 genes. However, they did not completely assemble the chromosomes, probably because of a limitation of the sequencing technology they used. In the current study, we were able to assemble all six individual chromosomes of M.pachydermatis with corresponding telomeres (Fig. 1). Most of the genome features of KCTC 27587 were similar to those of the type strain CBS 1879.

\section{M. pachydermatis KCTC 27587 Harbors a Tandem Quadruplication in Chromosome 4}

During the sequence assembly, we found that coverage of the region between the chromosomal positions 907,643 and 992,110 on chromosome 4 was $350-400 \times$, which was significantly higher than the average coverage $(97.76 \times)$ of

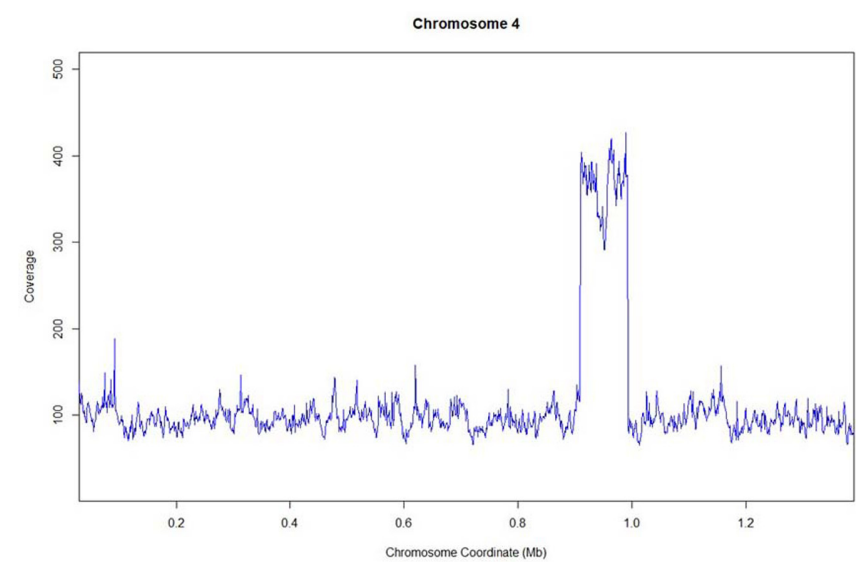

Fig. 2. Tandem quadruplication in chromosome 4.

The sequencing coverage of the region between positions 907,643 and 992,110 in chromosome 4 was $350-400 \times$, whereas the average of coverage of the genome was $97.76 \times$, suggesting that the region is tandemly quadruplicated.

the remainder of chromosome 4 and other chromosomes, suggesting that the region is tandemly quadruplicated (Fig. 2). In the quadruplicated region, 52 protein-coding genes were identified, including MP87_02948 and MP87_02952, homologs of S. cerevisiae ERG11 and ERG4, which encode lanosterol 14- $\alpha$ demethylase and sterol C-24 reductase, respectively, involved in the ergosterol biosynthesis pathway (Fig. 3). To confirm the existence of the tandemly quadruplicated region in chromosome 4, we determined the relative copy numbers of three genes (MP87_02934, MP87_02948, and MP87_02974, which are homologs of S. cerevisiae CCA1, ERG11, and PET8 respectively) in the quadruplicated region by Q-RT-PCR. The genes MP87_00784, MP87_01226, MP87_02511, and MP87_03488, which encode translation elongation factor 2, GAPDH, eIF-5 $\alpha$, and

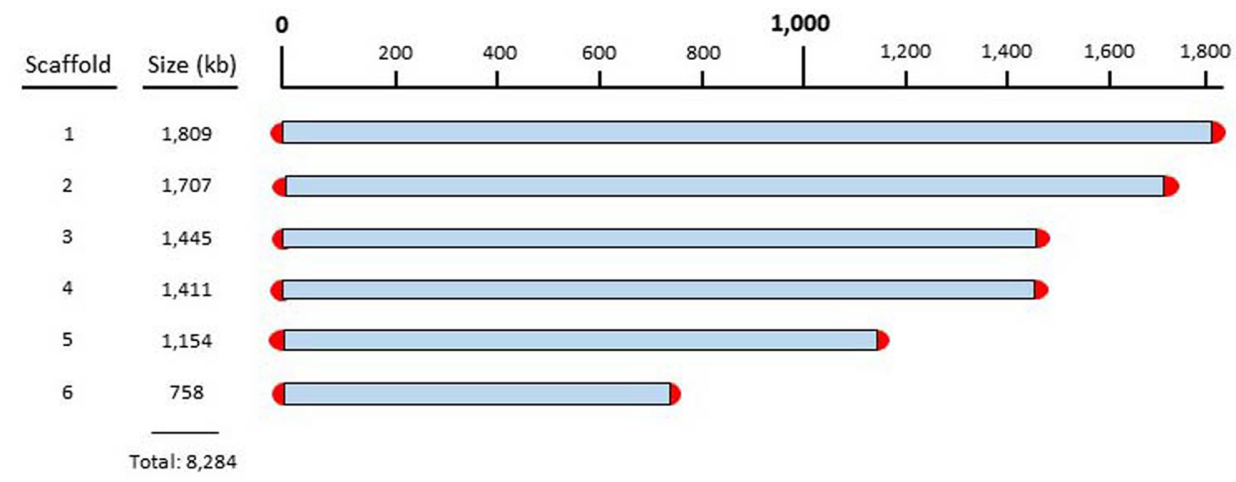

Fig. 1. M. pachydermatis KCTC 27587 has six chromosomes.

The scaffolds of more than $20 \mathrm{~kb}$ from M. pachydermatis KCTC 27587 represent the six chromosomes. Identified telomere repeats are indicated by red half-circles at the end of the scaffold. 


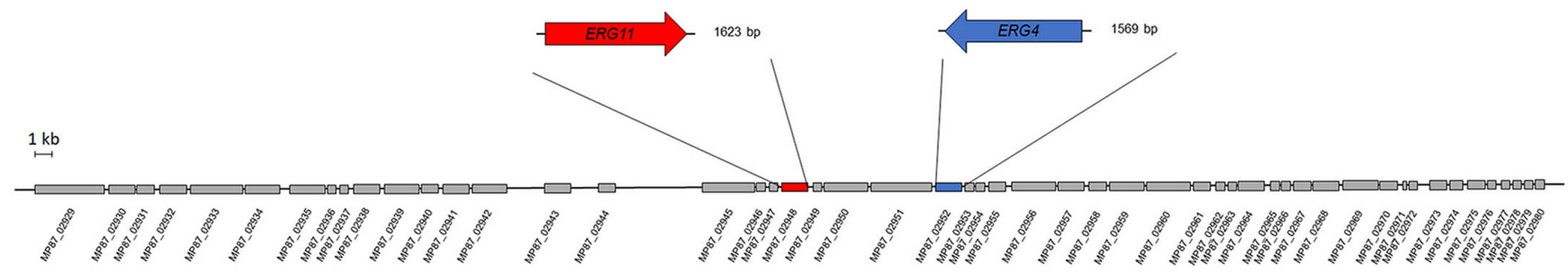

Fig. 3. Organization of the quadruplicated region.

In total, 52 protein-coding genes were found in the tandemly quadruplicated region, including homologs of S. cerevisiae ERG4 and ERG11, which encode sterol C-24 reductase and lanosterol 14- $\alpha$ demethylase, respectively.

$\alpha$-tubulin, respectively, located in a non-quadruplicated region, were used as references. The Q-RT-PCR results showed that the copy numbers of MP87_02934, MP87_02948, and MP87_02974 were approximately four times higher than those of the reference genes (Fig. 4). These data confirmed the existence of the tandemly quadruplicated region in chromosome 4 suggested by the whole genome sequencing data, and led us to hypothesize that the chromosomal rearrangement might be contributed to the significantly reduced ketoconazole susceptibility of $M$. pachydermatis KCTC 27587.

\section{Transcriptome Analysis}

Earlier studies in C. neoformans have shown that multiple duplication of a chromosome in azole-resistant strains results in increased expression of genes within the chromosome, although the level of increase may or may not correspond with the amount of duplication [28,29]. Therefore, we investigated whether the quadruplication in chromosome 4 influenced on the transcription of genes in that region. The transcriptome of M.pachydermatis KCTC 27587 was analyzed by RNA-sequencing and was compared with that of the type strain M. pachydermatis CBS 1879. Overall, the
A

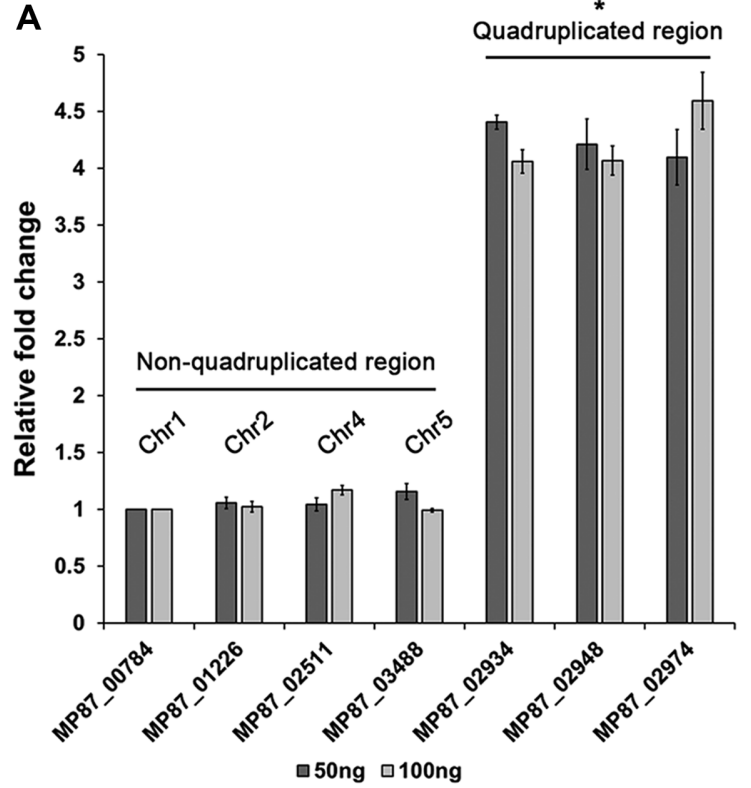

B

\begin{tabular}{ccccc}
\hline Non-quadruplicated region (reference) & & & \\
\hline Gene ID & Annotation & Chromosome & Start & End \\
\hline MP87_00784 & Translation elongation factor 2 (TEF2) & Chr1 & 1584810 & 1587278 \\
MP87_01226 & GAPDH & Chr2 & 672520 & 673624 \\
MP87_02511 & Translation initiation factor 5 (elF-5a) & Chr4 & 169179 & 170505 \\
MP87_03488 & a-tubulin & Chr5 & 543359 & 544714 \\
\hline Quadruplicated region & Annotation & Chromosome & Start & End \\
\hline Gene ID & tRNA nucleotidyltransferase CCA1 & Chr4 & 918687 & 920519 \\
\hline MP87_02934 & ERG11 & Chr4 & 949247 & 950869 \\
MP87_02948 & S-adenosylmethionine transporter PET8 & Chr4 & 986522 & 987291 \\
MP87_02974 & & & &
\end{tabular}

Fig. 4. Confirmation of gene copy numbers in the quadruplicated region.

(A) Results of Q-RT-PCR using genomic DNA extracted from M. pachydermatis KCTC 27587 as a template confirmed the existence of quadruplication in chromosome 4. Data were obtained from three independent experiments and an asterisk indicates statistical significance $(p<0.05)$. (B) Primers specific to MP87_02934 (CCA1), MP87_02948 (ERG11) and MP87_02974 (PET8) genes, which are located in the quadruplicated region, and MP87_00784 (TEF2), MP87_01226 (GAPDH), MP87_02511 (eIF-5 $\alpha$ ) and MP87_03488 ( $\alpha$-tubulin) genes, which are located in the non-quadruplicated region as references, were used for Q-RT-PCR. 


\begin{tabular}{|c|c|c|}
\hline Gene ID & Product & \\
\hline MP87_02929 & mef2-translation elongation mitochondrial & 1.89 \\
\hline MP87 02930 & hypothetical protein Malapachy 4038 & 2.13 \\
\hline MP87_02931 & hypothetical protein Malapachy_4026 & 2.34 \\
\hline MP87_02932 & hypothetical protein Malapachy_4039 & 5.01 \\
\hline MP87_02933 & mitogen-activated protein kinase & 2.12 \\
\hline MP87_02934 & trna nucleotidyltransferase & 2.12 \\
\hline MP87_02935 & hypothetical protein Malapachy_4048 & 4.10 \\
\hline MP87_02936 & hypothetical protein Malapachy_4048 & 2.15 \\
\hline MP87_02937 & hypothetical protein Malapachy_4059 & 6.12 \\
\hline MP87_02938 & peptidyl-prolyl cis-trans isomerase-like 2 & 1.95 \\
\hline MP87_02939 & peroxisomal targeting signal-1 receptor & 1.80 \\
\hline MP87_02940 & dhh phosphoesterase & 2.68 \\
\hline MP87_02941 & atp-dependent rna helicase dbpg & 4.42 \\
\hline MP87_02942 & hypothetical protein Malapachy_4033 & 3.95 \\
\hline MP87_02943 & ferric reductase & 13.15 \\
\hline MP87_02944 & ferric reductase & 120.34 \\
\hline MP87_02945 & hypothetical protein Malapachy_ 4036 & 3.94 \\
\hline MP87_02946 & purine nucleoside permease & 2.84 \\
\hline MP87_02947 & purine nucleoside permease & 8.65 \\
\hline MP87_02948 & cytochrome p450 & 3.68 \\
\hline MP87_02949 & hypothetical protein Malapachy_4046 & 17.55 \\
\hline MP87_02950 & hom6-homoserine dehydrogenase & 2.21 \\
\hline MP87_02951 & polyphosphoinositide phosphatase & 2.06 \\
\hline MP87_02952 & erg4 erg24 ergosterol biosynthesis protein & 2.81 \\
\hline MP87_02953 & 60 r ribosomal protein mitochondrial & 4.75 \\
\hline MP87_02954 & hypothetical protein Malapachy_4050 & 2.11 \\
\hline MP87_02955 & hypothetical protein Malapachy_4050 & 5.66 \\
\hline MP87_02956 & copper p-type atpase & 4.06 \\
\hline MP87_02957 & f-box cyclin-like domain containing protein & 1.77 \\
\hline MP87_02958 & hypothetical protein Malapachy_4045 & 2.96 \\
\hline MP87_02959 & flap endonuclease gen-like protein 1 . & 2.24 \\
\hline MP87_02960 & signal recognition particle protein & 2.48 \\
\hline MP87_02961 & glycosyltransferase family 76 protein & 4.39 \\
\hline MP87_02962 & hypothetical protein Malapachy_ 4056 & 2.34 \\
\hline MP87_02963 & ap-2 complex subunit sigma & 1.02 \\
\hline MP87_02964 & mitochondrion organization and biogenesis protein & 3.56 \\
\hline MP87_02965 & hypothetical protein Malapachy_4005 & 3.56 \\
\hline MP87_02966 & translocation protein sec72 & 1.96 \\
\hline MP87_02967 & atp12-domain-containing protein & 2.45 \\
\hline MP87_02968 & integral peroxisomal membrane protein & 2.78 \\
\hline MP87_02969 & $\begin{array}{l}\text { transmembrane protein subunit of the glycosylphosphatidylinositol transamidase complex }\end{array}$ & 3.84 \\
\hline MP87_02970 & hypothetical protein Malapachy_4058 & 2.58 \\
\hline MP87_02971 & hypothetical protein Malapachy_4060 & 2.36 \\
\hline MP87_02972 & sip1 domain-containing protein & 12.19 \\
\hline MP87_02973 & nop52-domain-containing protein & 4.26 \\
\hline MP87_02974 & s-adenosylmethionine transporter & 3.73 \\
\hline MP87_02975 & amidase signature enzyme & 4.07 \\
\hline MP87_02976 & amidase signature enzyme & 3.85 \\
\hline MP87_02977 & hypothetical protein Malapachy_4044 & 2.33 \\
\hline MP87_02978 & hypothetical protein Malapachy_4053 & 1.73 \\
\hline MP87_02979 & hypothetical protein Malapachy_4053 & 3.46 \\
\hline \multirow[t]{2}{*}{ MP87_02980 } & tpr-like protein & 4.17 \\
\hline & 1 & $>5>1$ \\
\hline
\end{tabular}

Fig. 5. Differential expression of genes in the quadruplicated region.

The transcriptome of M. pachydermatis KCTC 27587 was analyzed by RNA-sequencing and was compared with that of the M. pachydermatis reference strain CBS 1879. Differential expression of 52 genes in the quadruplicated region in chromosome 4 of $M$. pachydermatis KCTC 27587 is presented. The values in the right column indicate the fold changes in M. pachydermatis KCTC 27587 versus M. pachydermatis CBS 1879.

transcript levels of genes within the quadruplicated region were higher than those in the type strain: 45 out of 52 genes showed a more than two-fold increase (Fig. 5). Particular attention was paid to two genes, MP87_02948 and MP87_02952, the homologs of S. cerevisiae ERG11 and ERG4, because increased expression of genes in the ergosterol pathway contribute to azole resistance in various pathogenic fungi. For example, elevated mRNA levels of ERG4 and ERG11 have been observed in azoleresistant C. albicans clinical isolates [30]. As we expected, the transcript levels of the ERG11 and ERG4 homologs in the quadruplicated region in M. pachydermatis KCTC 27587 were significantly higher (3.68- and 2.81-fold, respectively) than those in the type strain. Considering that increased expression of ERG11 and ERG4 is one of the common mechanisms of azole resistance in other pathogenic fungi, we concluded that the overexpression of the ERG4 and ERG11 homologs, which may be caused by quadruplication of the genomic region, might be the main cause of ketoconazole resistance in M. pachydermatis KCTC 27587. We note that the genes encoding homologs of ferric reductases, MP87_02943 and MP87_02944, in the quadruplicated region were also more strongly expressed in M.pachydermatis KCTC 27587, which may also influence susceptibility of the yeast to ketoconazole as the enzyme has been shown to contribute to azole resistance [31, 32]. Transcript levels of two other genes in the quadruplicated region, encoding a hypothetical protein (MP87_02949) and the homolog of S. cerevisiae Sip1 domain containing protein (MP87_02972), respectively, were also significantly increased by $>10$-fold. However, the function of these genes in relation with azole resistance in fungi is unknown, and therefore, further investigation is required to define the contribution of these genes to ketoconazole resistance, although such contribution is unlikely.

\section{Chromosomal Rearrangement Occurs in Ketoconazole- Resistant M. pachydermatis Strains Generated by In Vitro Evolution}

We next questioned whether chromosomal rearrangement, such as the tandem quadruplication observed in the current study, commonly occurs in ketoconazole-resistant M. pachydermatis strains. To answer this question, we artificially generated ketoconazole-resistant strains by in vitro evolution using the ketoconazole-susceptible strain KCTC 27575, which was isolated from a healthy dog. In total, four independent resistant strains showing MICs equivalent to that of KCTC 27587 were obtained and were named "75IVE-1," “75IVE-2," “75IVE-3," and "75IVE-4." In parallel, the susceptible strain KCTC 27575 was periodically transferred to drug-free media. The reference strain generated in this way to observe any effect of spontaneous mutation was named "75IVE-C."

The copy numbers of the chromosomal region that is quadruplicated in M. pachydermatis KCTC 27587 in strains 75IVE-1, 75IVE-2, 75IVE-3, and 75IVE-4 were determined by Q-RT-PCR. We first determined the relative gene copy number of MP87_02948, the ERG11 homolog, within the quadruplicated region and found that two out of the four artificially-generated resistant strains, i.e., 75IVE-1 and 
A
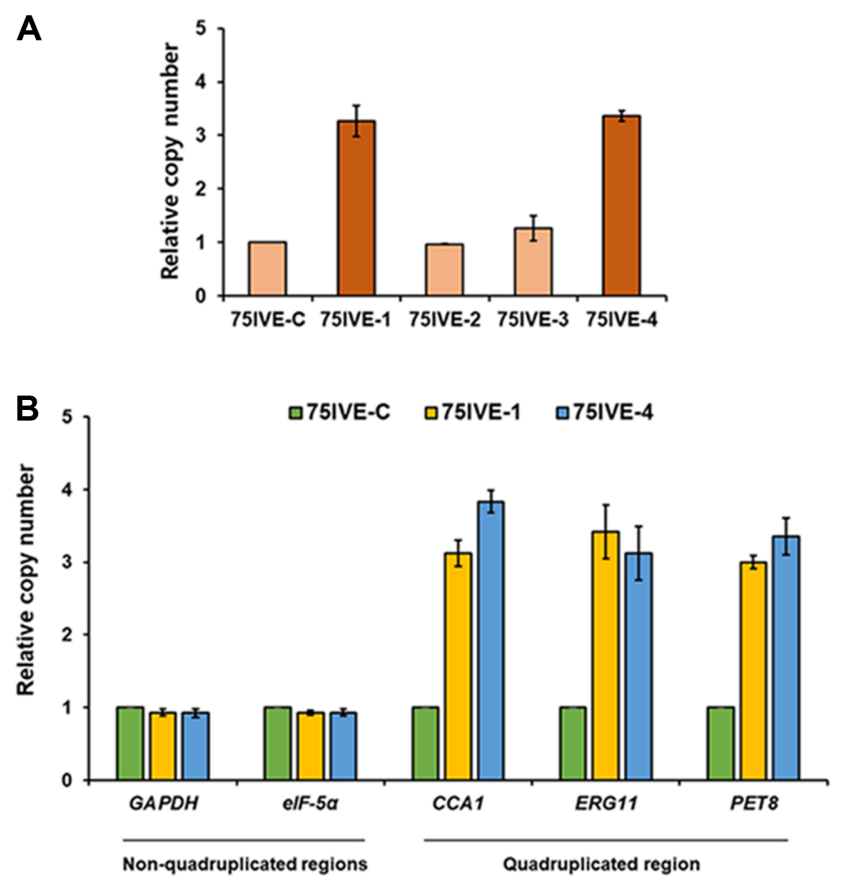

Fig. 6. Confirmation of genomic multiplication in ketoconazoleresistant strains generated by in vitro evolution.

(A) Four independent ketoconazole-resistant strains, 75IVE-1, 75IVE-2, 75IVE-3, and 75IVE-4, were artificially-generated by in vitro evolution. Two strains, 75IVE-1 and 75IVE-4, displayed more than four times higher copy numbers of the ERG11 homolog than the ketoconazolesusceptible parental strain, 75IVE-C (M. pachydermatis KCTC 27575). (B) Relative copy numbers of genes located in the potentially quadruplicated region in strains 75IVE-1 and 75IVE-4 were estimated by Q-RT-PCR. The relative copy numbers of the genes (CCA1, ERG11, and PET8 homologs) in the quadruplicated region were higher than those in the control strain, whereas the relative copy numbers of reference genes (GAPDH and eIF- $5 \alpha$ homologs) located in the nonquadruplicated regions were not different from those in the parental strain. Data were obtained from three independent experiments.

75IVE-4, showed more than three times higher copy numbers than the reference strain 75IVE-C (Fig. 6A). These results implied the existence of quadruplication in the chromosomal region containing MP87_02948 (ERG11) in strains 75IVE-1 and 75IVE-4. To confirm this, we designed primers targeting MP87_01226 (GAPDH) and MP87_02511 (eIF-5 $\alpha$ ) located in the non-quadruplicated region and used as reference genes, as well as primers targeting MP87_02934 (CCA1), MP87_02948 (ERG11), and MP87_02974 (PET8) located within the quadruplicated regions. As shown in Fig. 6B, the relative copy numbers of MP87_02934 (CCA1), MP87_02948 (ERG11), and MP87_02974 (PET8) were greater than those of the reference genes MP87_01226
(GAPDH) and MP87_02511 (eIF-5 $\alpha$ ), in strains 75IVE-1 and 75IVE-4 confirming the existence of multiplication of the chromosomal region. These results suggested that gene multiplication occurred in the artificially-generated ketoconazole-resistant $M$. pachydermatis strains.

Chromosomal rearrangements, such as duplication and translocation, often cause alteration of phenotypic characteristics in various organisms [33-35]. In fungi, numerous cases of chromosomal duplication and translocation as well as gene amplification have been observed and are considered mechanisms of resistance to growth inhibitors and abiotic stresses [33, 34, 36, 37]. It has been suggested that highly repeated sequences in chromosomes are involved in chromosomal duplication and translocation; however, the underlying mechanism remains to be elucidated.

In the current study, we found that M. pachydermatis underwent extensive chromosomal rearrangement, i.e., tandem quadruplication, to adapt to the pressure of ketoconazole, which has resulted in increased expression levels of the genes involved in the pathway targeted by the drug. Moreover, our in vitro evolution assay revealed that chromosomal rearrangement might be one of the strategies of $M$. pachydermatis to survive in the presence of high concentrations of ketoconazole.

\section{Acknowledgments}

This research was supported by the Chung-Ang University Graduate Research Scholarship in 2017 (YJC), and by the Basic Science Research Program through the National Research Foundation of Korea funded by the Ministry of Science, ICT and Future Planning NRF-2016R1D1A1B03931890 (WJ).

\section{Conflict of Interest}

The authors have no financial conflicts of interest to declare.

\section{References}

1. Hill PB, Lo A, Eden CA, Huntley S, Morey V, Ramsey S, et al. 2006. Survey of the prevalence, diagnosis and treatment of dermatological conditions in small animals in general practice. Vet. Rec. 158: 533-539.

2. Saridomichelakis MN, Farmaki R, Leontides LS, Koutinas AF. 2007. Aetiology of canine otitis externa: a retrospective study of 100 cases. Vet. Dermatol. 18: 341-347.

3. Korbelik J, Singh A, Rousseau J, Weese JS. 2018. Analysis of the otic mycobiota in dogs with otitis externa compared to 
healthy individuals. Vet. Dermatol. 29: 417-e138.

4. Negre A, Bensignor E, Guillot J. 2009. Evidence-based veterinary dermatology: a systematic review of interventions for Malassezia dermatitis in dogs. Vet. Dermatol. 20: 1-12.

5. Ilahi A, Hadrich I, Goudjil S, Kongolo G, Chazal C, Leke A, et al. 2018. Molecular epidemiology of a Malassezia pachydermatis neonatal unit outbreak. Med. Mycol. 56: 69-77.

6. Chryssanthou E, Broberger U, Petrini B. 2001. Malassezia pachydermatis fungaemia in a neonatal intensive care unit. Acta Paediatr. 90: 323-327.

7. Bajwa J. 2017. Canine Malassezia dermatitis. Can. Vet. J. 58: 1119-1121.

8. Cole LK, Luu DH, Rajala-Schultz PJ, Meadows C, Torres AH. 2007. In vitro activity of an ear rinse containing tromethamine, EDTA, benzyl alcohol and $0.1 \%$ ketoconazole on Malassezia organisms from dogs with otitis externa. Vet. Dermatol. 18: 115-119.

9. Yoshida Y, Aoyama Y. 1987. Interaction of azole antifungal agents with cytochrome P-45014DM purified from Saccharomyces cerevisiae microsomes. Biochem. Pharmacol. 36: 229235.

10. Truan G, Epinat JC, Rougeulle C, Cullin C, Pompon D. 1994. Cloning and characterization of a yeast cytochrome b5-encoding gene which suppresses ketoconazole hypersensitivity in a NADPH-P-450 reductase-deficient strain. Gene 142: 123-127.

11. Jesus FP, Lautert C, Zanette RA, Mahl DL, Azevedo MI, Machado ML, et al. 2011. In vitro susceptibility of fluconazole-susceptible and -resistant isolates of Malassezia pachydermatis against azoles. Vet. Microbiol. 152: 161-164.

12. Watanabe S, Koike A, Kano R, Nagata M, Chen C, Hwang CY, et al. 2014. In vitro susceptibility of Malassezia pachydermatis isolates from canine skin with atopic dermatitis to ketoconazole and itraconazole in East Asia. J. Vet. Med. Sci. 76: 579-581.

13. Iatta R, Puttilli MR, Immediato D, Otranto D, Cafarchia C. 2017. The role of drug efflux pumps in Malassezia pachydermatis and Malassezia furfur defence against azoles. Mycoses 60: 178-182.

14. Midgley G. 1989. The diversity of Pityrosporum (Malassezia) yeasts in vivo and in vitro. Mycopathologia 106: 143-153.

15. Walsh PS, Metzger DA, Higuchi R. 2013. Chelex 100 as a medium for simple extraction of DNA for PCR-based typing from forensic material. Biotechniques 54: 134-139.

16. Gupta AK, Boekhout T, Theelen B, Summerbell R, Batra R. 2004. Identification and typing of Malassezia species by amplified fragment length polymorphism and sequence analyses of the internal transcribed spacer and large-subunit regions of ribosomal DNA. J. Clin. Microbiol. 42: 4253-4260.

17. Wayne P. 2008. Clinical and Laboratory Standards Institute: Reference method for broth dilution antifungal susceptibility testing of yeasts; approved standard-; CLSI document M27-A3.

18. van Burik JA, Schreckhise RW, White TC, Bowden RA,
Myerson D. 1998. Comparison of six extraction techniques for isolation of DNA from filamentous fungi. Med. Mycol. 36: 299-303.

19. Koren S, Walenz BP, Berlin K, Miller JR, Bergman NH, Phillippy AM. 2017. Canu: scalable and accurate long-read assembly via adaptive k-mer weighting and repeat separation. Genome Res. 27: 722-736.

20. Hoff KJ, Lange S, Lomsadze A, Borodovsky M, Stanke M. 2016. BRAKER1: Unsupervised RNA-Seq-Based Genome Annotation with GeneMark-ET and AUGUSTUS. Bioinformatics 32: 767-769.

21. Huerta-Cepas J, Forslund K, Coelho LP, Szklarczyk D, Jensen LJ, von Mering C, et al. 2017. Fast genome-wide functional annotation through orthology assignment by eggNOG-mapper. Mol. Biol. Evol. 34: 2115-2122.

22. Bolger AM, Lohse M, Usadel B. 2014. Trimmomatic: a flexible trimmer for Illumina sequence data. Bioinformatics 30: 2114-2120.

23. Langmead B, Salzberg SL. 2012. Fast gapped-read alignment with Bowtie 2. Nat. Methods 9: 357-359.

24. Liao Y, Smyth GK, Shi W. 2014. Feature counts: an efficient general purpose program for assigning sequence reads to genomic features. Bioinformatics 30: 923-930.

25. Love MI, Huber W, Anders S. 2014. Moderated estimation of fold change and dispersion for RNA-seq data with DESeq2. Genome Biol. 15: 550.

26. Livak KJ, Schmittgen TD. 2001. Analysis of relative gene expression data using real-time quantitative PCR and the 2- $\Delta \Delta$ CT method. Methods 25: 402-408.

27. da Silva Ferreira ME, Capellaro JL, dos Reis Marques E, Malavazi I, Perlin D, Park S, et al. 2004. In vitro evolution of itraconazole resistance in Aspergillus fumigatus involves multiple mechanisms of resistance. Antimicrob. Agents Chemother. 48: 4405-4413.

28. Hu G, Wang J, Choi J, Jung WH, Liu I, Litvintseva AP, et al. 2011. Variation in chromosome copy number influences the virulence of Cryptococcus neoformans and occurs in isolates from AIDS patients. BMC Genomics 12: 526.

29. Sionov E, Lee H, Chang YC, Kwon-Chung KJ. 2010. Cryptococcus neoformans overcomes stress of azole drugs by formation of disomy in specific multiple chromosomes. PLoS Pathog. 6: e1000848.

30. Feng W, Yang J, Xi Z, Qiao Z, Lv Y, Wang Y, et al. 2017. Mutations and/or overexpressions of ERG4 and ERG11 genes in clinical azoles-resistant isolates of Candida albicans. Microb. Drug Resist. 23: 563-570.

31. Gerwien F, Safyan A, Wisgott S, Brunke S, Kasper L, Hube B. 2017. The fungal pathogen Candida glabrata does not depend on surface ferric reductases for iron acquisition. Front. Microbiol. 8:1055.

32. Saikia S, Oliveira D, Hu G, Kronstad J. 2014. Role of ferric reductases in iron acquisition and virulence in the fungal pathogen Cryptococcus neoformans. Infect. Immun. 82: 839-850. 
33. Koszul R, Caburet S, Dujon B, Fischer G. 2004. Eucaryotic genome evolution through the spontaneous duplication of large chromosomal segments. EMBO J. 23: 234-243.

34. Koszul R, Dujon B, Fischer G. 2006. Stability of large segmental duplications in the yeast genome. Genetics 172: 2211-2222.

35. McBride DJ, Etemadmoghadam D, Cooke SL, Alsop K, George J, Butler A, et al. 2012. Tandem duplication of chromosomal segments is common in ovarian and breast cancer genomes. J. Pathol. 227: 446-455.

36. Mehra RK, Garey JR, Winge DR. 1990. Selective and tandem amplification of a member of the metallothionein gene family in Candida glabrata. J. Biol. Chem. 265: 6369-6375.

37. Aladjem MI, Koltin Y, Lavi S. 1988. Enhancement of copper resistance and CupI amplification in carcinogen-treated yeast cells. Mol. Gen. Genet. 211: 88-94. 